\title{
Pharmacologic Substance
}

National Cancer Institute

\section{Source}

National Cancer Institute. Pharmacologic Substance. NCI Thesaurus. Code C1909.

Any natural, endogenously-derived, synthetic or semi-synthetic compound with

pharmacologic activity. A pharmacologic substance has one or more specific mechanism

of action(s) through which it exerts one or more effect(s) on the human or animal body.

They can be used to potentially prevent, diagnose, treat or relieve symptoms of a

disease. Formulation specific agents and some combination agents are also classified as pharmacologic substances. 